\title{
Elevator Group Control System Based on Fuzzy Reasoning
}

Chen $\mathrm{KE}^{1 *}$, Chen Bin ${ }^{1}$, Wang Jian-rong ${ }^{1}$

${ }^{1}$ School of Information Engineering, Shenyang University of Chemical Technology, Shenyang, Liaoning 110142

DOI: $10.36347 /$ sjet.2022.v10i01.001

| Received: 17.12.2021 | Accepted: 21.01.2022 | Published: 24.01.2022

*Corresponding author: Chen KE

School of Information Engineering, Shenyang University of Chemical Technology, Shenyang, Liaoning 110142

\section{Abstract}

Review Article

Due to the characteristics of the elevator system itself, traditional control algorithms are difficult to apply to elevator dispatching. However, the fuzzy control algorithm does not require the establishment of an accurate mathematical model, and can be well integrated with the elevator system. Based on fuzzy control, the elevator dispatch can be optimized. The traffic fuzzy inference controller is designed to identify the elevator traffic mode according to the flow of people; the elevator fuzzy inference device is designed to construct the objective function according to the evaluation index. Siemens S7-1200 series PLC is used to control the elevator, the elevator model uses Elevator Simulation Pro for simulation debugging, and Portal WINCC is used as the host computer for debugging to monitor.

Keywords: Fuzzy control; Elevator group control system; PLC S7-1200; Traffic recognition; Scheduling optimization.

Copyright (C) 2022 The Author(s): This is an open-access article distributed under the terms of the Creative Commons Attribution 4.0 International License (CC BY-NC 4.0) which permits unrestricted use, distribution, and reproduction in any medium for non-commercial use provided the original author and source are credited.

\section{INTRODUCTION}

Elevators are non-linear, discrete and uncertain, so it is difficult to establish an accurate mathematical model [1]. The fuzzy control algorithm is based on the expert experience to establish a simple reasoning model. The characteristic of fuzzy control is that there is no precise requirement for the model of the control system, and it has strong robustness to the linear and nonlinear parameter changes. Therefore, the fuzzy control Algorithms can be applied to elevator systems and improve the overall performance of the system. In this paper, a fuzzy control algorithm is used to construct two fuzzy inferencers to optimize and dispatch the group control system. The traffic mode fuzzy inferencer will detect the flow of people and infer the current traffic mode. Different traffic modes have different weights. Different performances are emphasized in the dispatch strategy. The dispatch strategy fuzzy reasoner infers the evaluation function value according to the control rules, and dispatches the ladder after constructing the optimal value of the objective function value.

The system is an elevator group control system based on fuzzy inference. The system is mainly composed of an upper computer and a lower computer. The upper computer uses WINCC for monitoring and arithmetic processing. The lower computer is composed of S7-1200PLC and elevator simulation software
Elevator Simulation Pro [2]. The overall system is composed of signal distribution module, data management module, traffic reasoning module and elevator control and elevator group modules [3]. The elevator group is composed of PLC and elevators; the signal distribution module transmits the signals of the elevator group and the control system; the data management module processes the system data; the traffic reasoning module is responsible for identifying the traffic mode of the system; the elevator control module is responsible for the overall dispatching and dispatching of the system [4].

\section{Fuzzy control}

The elevator group control system selects the dispatching plan according to the change of the human flow in the building. This paper designs a fuzzy reasoner to identify the current elevator traffic mode based on the elevator human flow conditions [5]. The fuzzy reasoner chooses to enter passengers within 5 minutes, leave passengers and the proportion of interfloor passengers, and divide the traffic mode into three major categories, namely the upward peak mode, the downward peak mode and the inter-floor mode.

Input parameter fuzzification is the fuzzy description of the controller input, establishing its universe and fuzzy set to describe the degree of membership [6], the fuzzy set of the system can use 
Chen KE et al., Sch J Eng Tech, Jan, 2022; 10(1): 1-4

small, medium, large To represent the flow of people, set $\mathrm{T}_{f}$ as the output of traffic recognition, $\mathrm{T}_{s}$ represents the up peak, $\mathrm{T}_{x}$ represents the down peak, and $\mathrm{T}_{c}$ represents the inter-layer mode.

Table-1: Fuzzy Set Membership Degree of Passenger Percentage

\begin{tabular}{|l|l|l|l|l|l|l|l|l|l|l|l|}
\hline $\begin{array}{l}\Omega_{r} \\
\Omega_{c} \\
\Omega_{l}\end{array}$ & 0 & $10 \%$ & $20 \%$ & $30 \%$ & $40 \%$ & $50 \%$ & $60 \%$ & $70 \%$ & $80 \%$ & $90 \%$ & $100 \%$ \\
\hline $\mathrm{L}$ & 1 & 0.8 & 0.6 & 0.3 & 0 & 0 & 0 & 0 & 0 & 0 & 0 \\
\hline $\mathrm{M}$ & 0 & 0 & 0 & 0.3 & 0.8 & 1 & 0.8 & 0.3 & 0 & 0 & 0 \\
\hline $\mathrm{H}$ & 0 & 0 & 0 & 0 & 0 & 0 & 0 & 0.3 & 0.6 & 0.8 & 1 \\
\hline
\end{tabular}

According to the above fuzzy set table, it can be seen that the system has 27 rules, combined with expert experience and only six rules are needed to realize the functions of the system. The traffic recognition controller and control rules are as follows.

(1) IF $\Omega_{r}=\mathrm{H}$ and $\Omega_{c}=\mathrm{L}$ and $\Omega_{l}=\mathrm{L}$ then $\mathrm{T}_{f}=\mathrm{T}_{s}$

(2) IF $\Omega_{r}=\mathrm{M}$ and $\Omega_{c}=\mathrm{L}$ and $\Omega_{l}=\mathrm{L}$ then $\mathrm{T}_{f}=\mathrm{T}_{s}$

(3) IF $\Omega_{r}=\mathrm{L}$ and $\Omega_{c}=\mathrm{H}$ and $\Omega_{l}=\mathrm{L}$ then $\mathrm{T}_{f}=\mathrm{T}_{x}$

(4) IF $\Omega_{r}=\mathrm{L}$ and $\Omega_{c}=\mathrm{M}$ and $\Omega_{l}=\mathrm{L}$ then $\mathrm{T}_{f}=\mathrm{T}_{x}$

(5) IF $\Omega_{r}=\mathrm{L}$ and $\Omega_{c}=\mathrm{L}$ and $\Omega_{l}=\mathrm{H}$ then $\mathrm{T}_{f}=\mathrm{T}_{c}$

6) IF $\Omega_{r}=\mathrm{L}$ and $\Omega_{c}=\mathrm{L}$ and $\Omega_{l}=\mathrm{M}$ then $\mathrm{T}_{f}=\mathrm{T}_{c}$

Fuzzy dispatching strategy is to calculate by evaluating indicators and traffic weights, and get the

$$
\mathrm{EZT}=\sum_{i=0}^{n} T_{a i}+\sum_{i=0}^{n} T_{a s i}+\sum_{i=0}^{n} T_{v i}+\sum_{i=0}^{n} T_{o t i}
$$
function value. evaluation index value in the dispatch strategy.

EZT

EXT

best dispatching plan by obtaining the largest objective

The input value of the elevator evaluation index[7] selects elevator calling time (EZT), elevator response time (EXT), long waiting (LWT), path concentration (RJD) and elevator capacity (ECV), and the evaluation index input value enters Output

Elevator call time refers to the time required for the elevator to reach the response floor from the current floor when the passenger presses the call button.

The time required for the elevator from the current signal to the last response signal is the elevator response time.

$$
E X T=\sum_{i=0}^{n} T_{s l i}
$$

(3) LWT

Long waiting refers to the time when passengers waiting and boarding exceeds a certain range value.

(4) RJD response floor.

Elevator path concentration refers to the difference between the height of the floor and the height of the

$$
\text { RJD }=\frac{|h 1-h 2| h}{H}
$$

\section{ECV}

Elevator capacity describes the response capacity of the elevator to new instructions.

$$
E C V=\frac{x_{i}}{\sum_{i=0}^{n} x_{i}}
$$

The most basic feature of fuzzy reasoning is to express the input value in a fuzzy input language, and then infer the output value through the expression of "IF...THEN", and finally de-fuzzify the output value and send it to the controlled object. The four evaluation indicators are represented by five fuzzy sets "VL", "L", "M", "H" and "VH".

The fuzzy rules of ART are determined by the two input variables of LWT and EXT. The smaller the 
Table-2: ART fuzzy rules

\begin{tabular}{|c|c|c|c|}
\hline$S_{\text {LWT }}$ EXT & $\mathbf{L}$ & $\mathbf{M}$ & $\mathbf{H}$ \\
\hline $\mathrm{L}$ & $\mathrm{VH}$ & $\mathrm{H}$ & $\mathrm{M}$ \\
\hline $\mathrm{M}$ & $\mathrm{H}$ & $\mathrm{L}$ & VL \\
\hline $\mathrm{H}$ & $\mathrm{M}$ & VL & VL \\
\hline
\end{tabular}

AWT is determined by the two inputs of EZT and EJD. When the route is optimal and the call time is shortest, the passenger takes the elevator to the destination in a very short time.

Table-3: AWT fuzzy rules

\begin{tabular}{|l|l|l|l|}
\hline \multicolumn{1}{|r|}{$S_{A W T}$ EXT } & \multirow{2}{*|}{ L } & M & H \\
\cline { 1 - 4 } & & & \\
\hline $\mathrm{L}$ & $\mathrm{H}$ & $\mathrm{M}$ & $\mathrm{VL}$ \\
\hline $\mathrm{M}$ & $\mathrm{VH}$ & $\mathrm{H}$ & $\mathrm{L}$ \\
\hline $\mathrm{H}$ & $\mathrm{H}$ & $\mathrm{L}$ & $\mathrm{VL}$ \\
\hline
\end{tabular}

PSR is determined by the three factors of ECV, EJD, and LWT. Therefore, there are 27 control rules for PSR. When the elevator capacity and longterm waiting are at $\mathrm{L}$, the elevator path aggregation degree is at L or M. Passenger satisfaction is better; when the elevator capacity and long-term waiting are at $M$ or above, the higher the elevator path aggregation degree, the passengers The worse the satisfaction.

RPC is determined by two fuzzy input parameters, EXT and ECV. The values of these two parameters directly affect the energy consumption of the elevator. When the response signal and the elevator capacity are small, the energy consumption is relatively low. The system will allocate elevators according to the energy consumption. When there are many response signals, the energy consumption of the elevator will increase, the value of $S_{R P C}$ will decrease, and the elevator priority will be the signal is allocated to the elevator with a larger $S_{R P C}$ value.

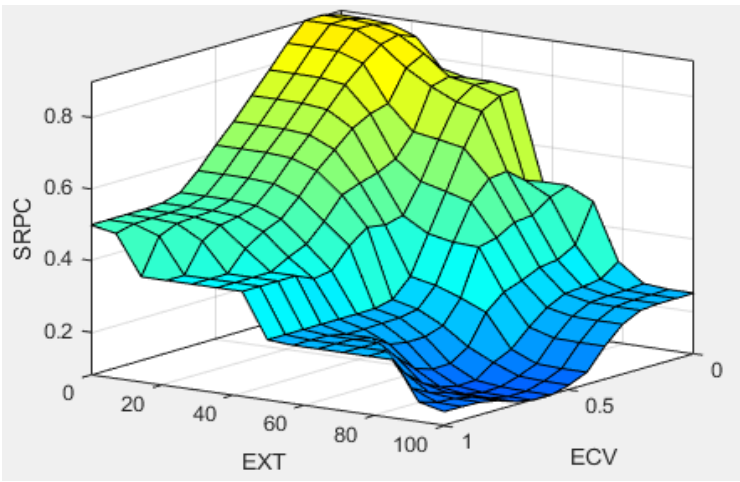

Fig-1: $S_{R P C}$ membership degree simulation

The input of the evaluation function passes through the fuzzy inference device of the dispatch strategy and outputs the fuzzy value of the evaluation function. The combination of the evaluation index and the corresponding traffic weight constitutes the dispatch objective function [8], and the objective function selects $S_{x}$ to dispatch the ladder.

$$
\begin{gathered}
S_{i}=\omega_{A R T} S_{A R T i}+\omega_{A W T} S_{A W T i}+\omega_{P S R} S_{P S R i}+\omega_{R P C} S_{R P C i} \\
S_{x}=\operatorname{MAX}\left\{S_{1}, S_{2}, S_{3}\right\}
\end{gathered}
$$

\section{Simulation}

After the communication between the upper computer and the lower computer is established, the elevator simulation EET is used for debugging. The verification result shows that the passenger arrival rate within 30 minutes is $15 \%$ higher than that of ordinary group control scheduling. Then, the fuzzy control algorithm is used to distinguish the flow of people. The dispatching objective function constructed by the combination of the transportation mode and the evaluation index can achieve dispatch optimization. It can be observed in EET that the energy consumption rate is reduced by about $10 \%$ compared with traditional scheduling. This is the fuzzy control rule for scheduling optimization to reduce the power consumption of sending multiple elevators to the target floor at the same time, so the fuzzy control algorithm is used to achieve the reduction the purpose of passenger time and energy consumption. 

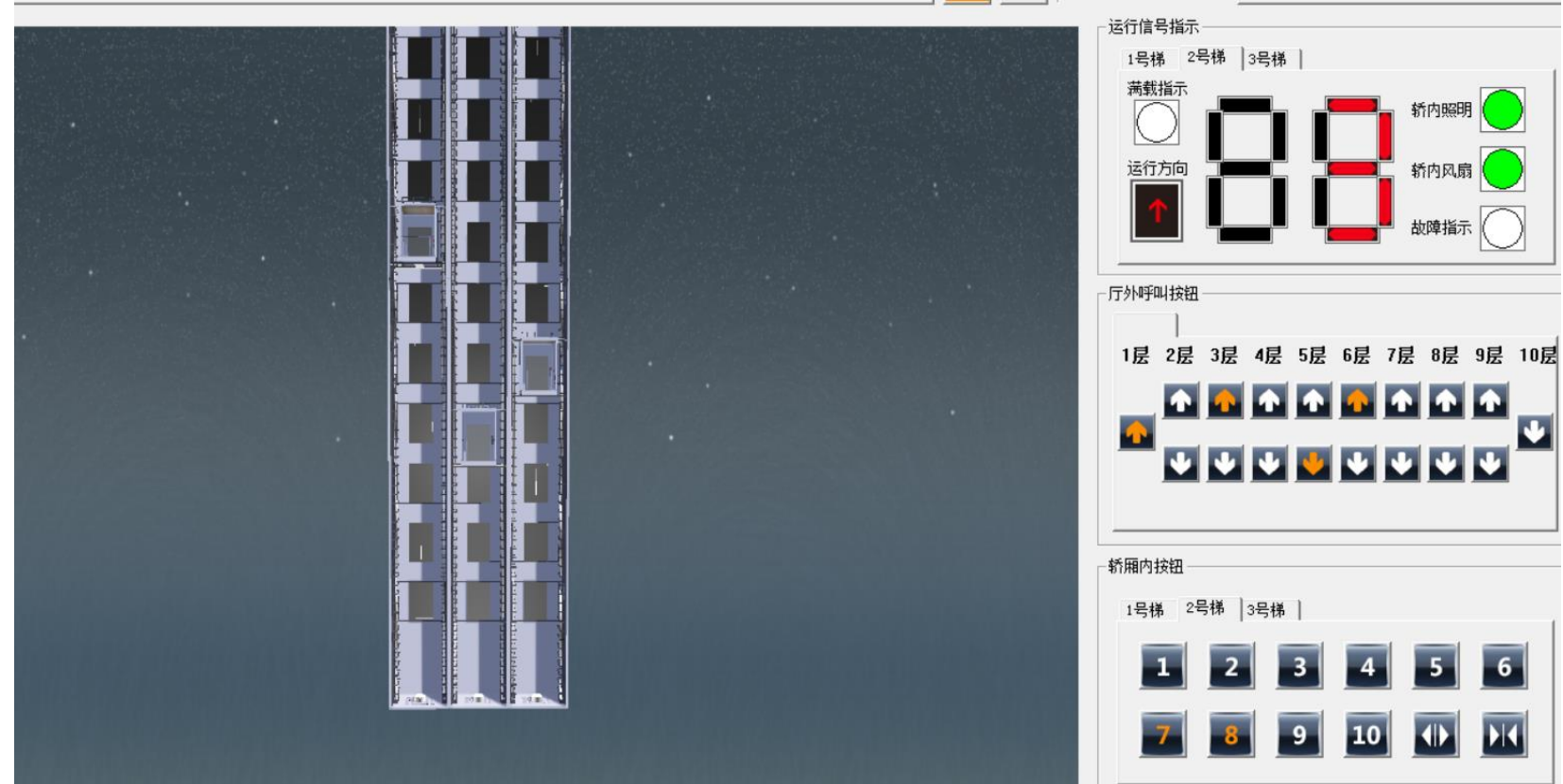

轿厢内按钮

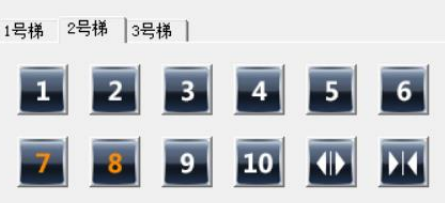

Fig-2: Elevator simulation diagram

\section{SUMMARIZE}

In this paper, fuzzy control algorithm is used to solve the problem of difficult to establish accurate elevator mathematical model, and the evaluation index and traffic mode are used to construct the objective function, and the elevator dispatching strategy is carried out according to the maximum objective function of each elevator. Adding the fuzzy control algorithm to the elevator group control system can effectively select different modes according to the flow of people, and achieve the purpose of scheduling optimization and energy saving.

\section{REFERENCES}

1. Zhang, F. (2020). Research and system implementation of elevator group control strategy based on fuzzy inference [D]. Taiyuan University of Science and Technology.

2. Liu, L. (2020). Group control elevator design based on PLC [D]. East China Jiaotong University.

3. Lan, Q. (2016). Research on Optimal Scheduling of
Elevator Group Control [D]. Taiyuan University of Technology.

4. Xu, W. (2017). Research on elevator group control method based on fuzzy control technology [D]. Jilin University.

5. Zeng, G. (2009). Design of elevator group control system based on fuzzy control technology [J]. Journal of Zhejiang University of Technology, $37(01) ; 115-118$.

6. Xue, X. (2019). Research on optimization algorithm of elevator group control system [D]. Xi'an Polytechnic University.

7. Liu, Q., Guan, Y. (2018). Elevator group control system based on fuzzy control [J]. Journal of North China University of Technology (Natural Science Edition), 40(01):104-112.

8. Zhang, W., Yang, J., Yang, B. (2019). Algorithm analysis of multi-objective elevator group control system based on S7-1200 PLC [J]. Mechatronic Engineering Technology, 48(08):36-37+70. 